\title{
The Recurrent Nova T Pyxidis - A Review
}

\section{Şölen Balman*}

Middle East Technical University, Ankara, Turkey

E-mail: solen@astroa.physics.metu.edu.tr

T Pyx is one of the most famous Galactic recurrent novae with puzzling characteristics that still needs to be resolved. Though T Pyx is a Cataclysmic Variable (CV) with an unusually high accretion rate $\left(\geq 10^{-8} \mathrm{M}_{\odot} \mathrm{yr}^{-1}\right)$ below the period gap showing recurrent nova outbursts, $\mathrm{T}$ Pyx portrays to be a moderately fast classical nova that ejects about $3 \times 10^{-5} \mathrm{M}_{\odot}$ in very eruption with about 20 to $40 \mathrm{yr}$ recurrence time. The mass of the WD in the system is $0.7-1.0 \mathrm{M}_{\odot}$ which is not the expected near-Chandrasekhar WD in recurrent nova systems postulated by the standard nova theory. In this paper, I review binary characteristics of T Pyx along with the detailed multiwavelength observations of the latest 2011 outburst of the system.

Standard disk theory at steady state predicts, an optically thick boundary layer at the accretion rate of quiescent $\mathrm{T}$ Pyx, with a blackbody emission in the soft X-ray/EUV regime. I review here that there is no such blackbody emission detected from T Pyx in the X-ray wavelengths and the quiescent Chandra observations (two months prior to outburst) are consistent with a multi-temperature thermal plasma emission model at a maximum temperature $k T_{\max }>47.0 \mathrm{keV}(2 \sigma$ lower limit is $37 \mathrm{keV}$ ). Thus, the $\mathrm{X}$-ray emission indicates that $\mathrm{T}$ Pyx has an optically thin boundary layer merged with an advection-dominated accretion Flow and/or X-ray corona in the inner disk indicating ongoing quasi-spherical accretion at (very) high rates during quiescent phases. Such a boundary layer structure may be excessively heating the white dwarf via advective hot accretion flows, influencing the thermonuclear runaway leading to the recurrent nova events.

In addition, I elaborate on the models of the circumstellar interaction of T Pyx outbursts and the existing old remnant. Balman (2014) shows detailed work on deconvolving the central source and its spectrum from any possible extended emission using high resolution imaging at the subpixel level revealing an extended emission with $\mathrm{S} / \mathrm{N} \sim 6-10$. The derived shape looks like an elliptical nebula with a semi-major axis $\sim 1.0$ arc sec. The nebulosity seems consistent with an interaction of the outflow/ejecta from the 1966 outburst.

The Golden Age of Cataclysmic Variables and Related Objects - III, Golden2015

7-12 September 2015

Palermo, Italy

\footnotetext{
* Speaker.
} 


\section{Introduction}

A cataclysmic variable (CV) is a close interacting binary system in which a white dwarf (WD) accretes material from its late-type low-mass main-sequence companion (Warner 1995). In nonmagnetic CVs the disk is believed to reach all the way to the WD (T Pyx is known to belong to this class); the material in the inner disk dissipates its kinetic energy in order to accrete onto the slowly rotating WD creating a boundary layer (BL) which is the transition region between the disk and the WD. Standard accretion disk theory predicts that half of the accretion luminosity originates from the disk in the optical and ultraviolet (UV) wavelengths and the other half emerges from the boundary layer as hard X-ray or extreme UV (EUV)/soft X-ray emission which can be summarized as $L_{\mathrm{BL}} \sim L_{\mathrm{disk}}=\mathrm{GM}_{\mathrm{WD}} \dot{M}_{\mathrm{acc}} / 2 \mathrm{R}_{\mathrm{WD}}=L_{\mathrm{acc}} / 2$ (Lynden-Bell \& Pringle 1974).

Classical (CN) and recurrent (RN) nova outbursts occur as a result of thermonuclear runaways (i.e., explosive ignition of accreted material) on the surface of the WD primaries in CV systems ejecting material in the range $10^{-7}$ to $10^{-3} \mathrm{M}_{\odot}$ with velocities from several hundred to several thousand kilometers per second (Shara 1989, Bode \& Evans 2008 and references therein). Recurrent nova outbursts occur with intervals of several decades. Nova outbursts show two main components of X-ray emission, a soft component dominating below $1 \mathrm{keV}$ originating from the hot post-outburst WD and a hard component emitting above $\sim 1 \mathrm{keV}$ as a result of accretion, windwind, and/or blast wave interaction (Krautter 2008). In CN and RN systems, the hard X-rays are mainly caused by the shocked plasma emission having plasma temperatures generally in a range 0.1-10 keV with luminosities $\leq \mathrm{a}$ few $\times 10^{36} \mathrm{erg} \mathrm{s}^{-1}$ in the outburst stage (Orio et al. 2001, Bode et al. 2006, Hernanz \& Sala 2002, Ness et al. 2009; Page et al. 2010, Nelson et al. 2012, Page et al. 2015).

The recurrent Nova T Pyx has an inter outburst time of $19 \pm 5.3$ yrs (Webbink et al. 1987) with outbursts in 1890, 1902, 1920, 1944, 1966. It shows remarkably similar light curves in the observed outbursts (see Figure 1). The nova is considered to be moderately fast with $t_{2}=31.6 \mathrm{~d}$ and $t_{3}=62 \mathrm{~d}$ (Schaefer 2010). A recent delayed outburst occurred in April 14, 2011 (Waagan et al. 2011) which was observed over the entire Electromagnetic Spectrum, particularly in the early rise to maximum phase.

\section{T Pyxidis - The Nova}

Ground-based optical imaging of the shell of T Pyx yields expansion speeds of 300-500 km/s (Shara et al. 1989; O'Brien \& Cohen 1998). HST Observations (1994-2007) indicate that the shell shows thousands of knots in $\mathrm{H} \alpha$ \& [NII]. The expansion velocities of these knots are $500-715 \mathrm{~km} / \mathrm{s}$ and they are not decelerated. The size of the main shell is $5 \mathrm{arcsec}$ and some faint emission out to 10 arcsec is also suggested (Shara et al. 1997; Schaefer, Pagnotta \& Shara 2010). The recent analysis by Schaefer et al. (2010) reveals a possible classical nova outburst in 1866.

\subsection{The 2011 outburst of T Pyxidis}

\subsubsection{Transient reflection nebula and the flash-ionized old ejecta}

A light echo was detected in the pre-existing clumpy ring with a radius of 5 arc sec and inclination angle of 30-40 degrees. The eastern edge of the reflecting ring is towards the observer. 

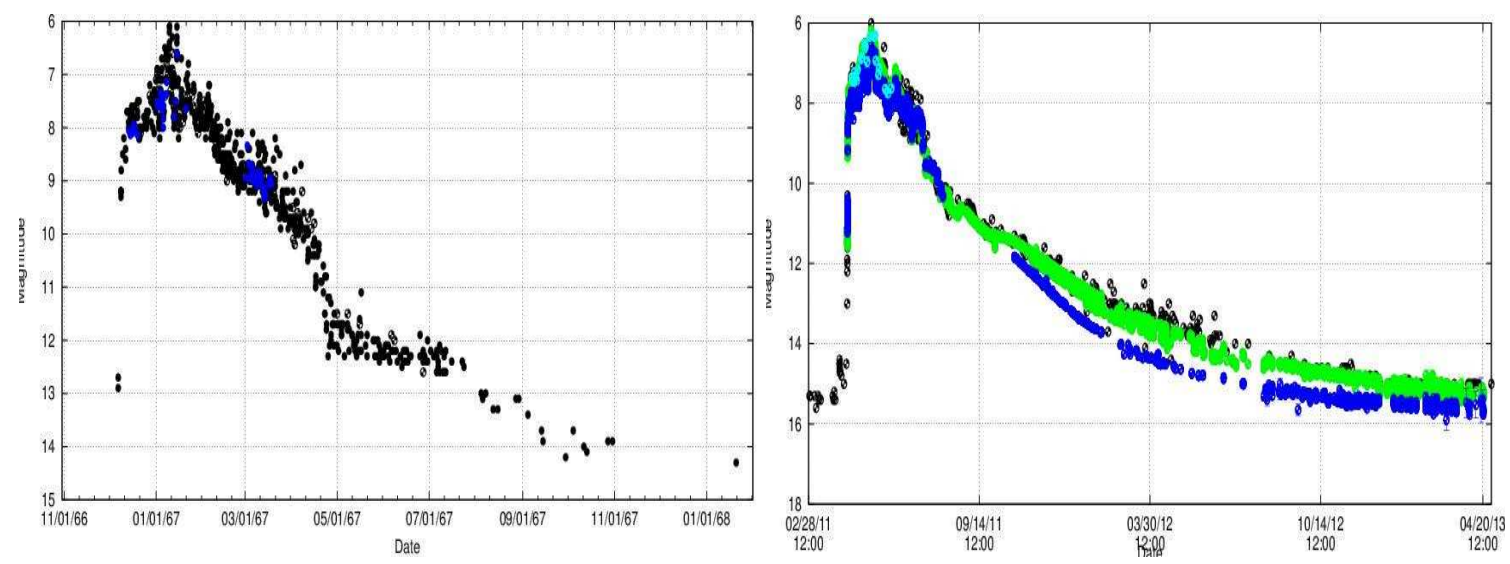

Figure 1: The AAVSO light curves of T Pyx compiled for the year 1966 on the right hand panel and 2011 on the left hand panel. Note the similarity of the light curves.

A distance of $4.8 \pm 0.5 \mathrm{kpc}$ is estimated using the size of the detected rings/arcs and assuming that the changes in the nebular echo brightness occur with the speed of light (see Sokoloski et al. 2013 for details).

Shara et al. (2015) studied the flash-ionized old ejecta using HST imaging during the 2011 outburst. The flash-ionized old ejecta is displayed in Figure 2 and indicate N-S photoionization of $\mathrm{H}$ (in color blue) during day 132 after outburst and NW-SW (in color red) at around day 183. The imaging analysis suggest a $\mathrm{H}$ density of at least $7 \times 10^{5} \mathrm{~cm}^{-3}$. There seems to be a large reservoir of old cold unseen diffuse $\mathrm{H}$ around (in the vicinity of) the [NII] knots. No $\mathrm{H}$ reservoir is found outside the oldest eruption region, thus showing no previous outbursts prior to 1866.
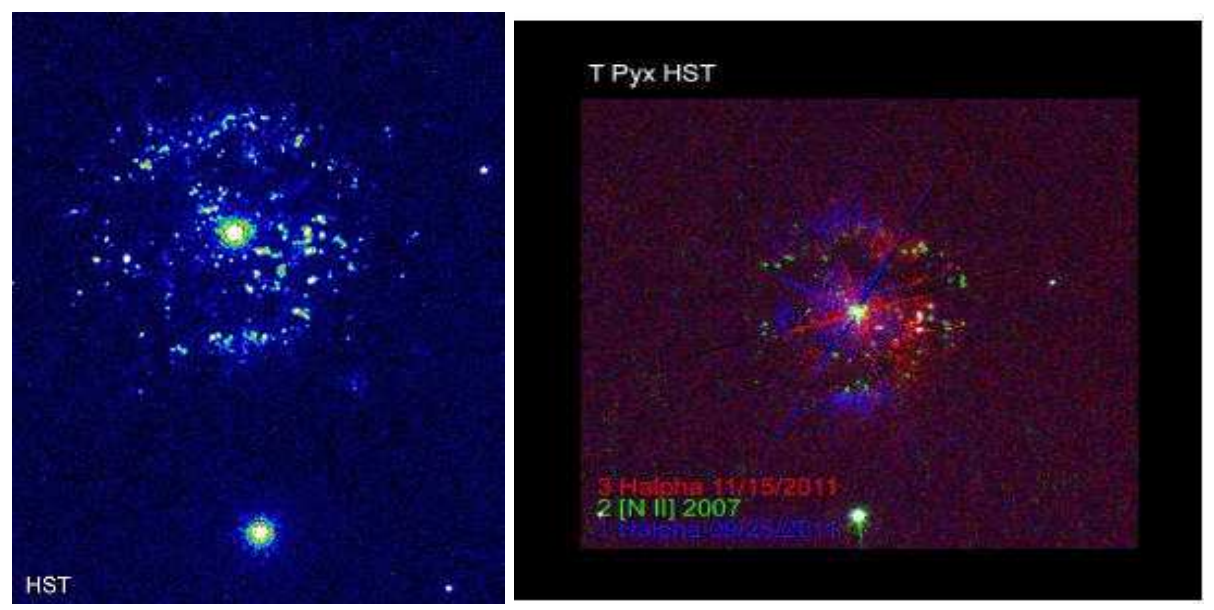

Figure 2: The left hand panel is the HST image of the old remnant of T Pyx. The right hand panel shows the ionization nebula detected using HST observations during the 2011 outburst (Shara et al. 2015).

\subsubsection{Optical, UV, and IR observations}

The SMEI optical light curve, provides detailed photometry in a time scale $t=1.5-49$ days post-discovery, and can be divided into four phases based on the idealized nova optical light curve: 
the initial rise (1.5-3.3 days), the pre-maximum halt (3.3-13.3 days), the final rise (14.7-27.9 days), and the early decline (27.9+ days) (Surina et al. 2014). A periodicity of $1.44 \pm 0.05 \mathrm{~d}$ was recovered during this phase. After $27.9 \mathrm{~d}$ an early decline is observed (Surina et al. 2014). During the rise phase, high velocity ejection out to $4000 \mathrm{~km} \mathrm{~s}^{-1}$ is detected which dropped at $5.7 \mathrm{~d}$ and afterwards stabilized around $1500 \mathrm{~km} \mathrm{~s}^{-1}$ with a higher mass-loss phase (Surina et al. 2014: SMARTS,LT; Izzo et al. 2012: VLT,TNG). In the early phases, T Pyx behaves as a typical classical nova, that has a photometric behavior consistent with an evolving pseudo-photosphere. Before maximum the nova shows broad prominent emission lines and P Cygni profiles. The $\mathrm{H} \alpha$ lines indicate $2200 \mathrm{~km}$ $\mathrm{s}^{-1}$ expansion velocity (Imamura \& Tanabe 2012). T Pyx portrays a hybrid nova where in the first 50 days, it is a He/N and afterwards an Fe II subclass (Izzo et al. 2012, Ederoclite 2014). Furthermore in the early phases interstellar absorption lines of $\mathrm{NaI}, \mathrm{CaII}, \mathrm{CH}, \mathrm{CH}^{+}$are detected with velocities in a range 11-80 $\mathrm{km} \mathrm{s}^{-1}$ (DACs) revealing circumstellar material around the nova (Shore et al. 2011).

In the following Fireball stage, emission lines of HeI HeII CIII NIII and P Cygni profies in the Balmer lines and HeI are measured out to $2500 \mathrm{~km} \mathrm{~s}^{-1}$. These lines are not detectable after $12 \mathrm{~d}$. Considering recombination effects, the mass of the ejecta is estimated as $10^{-5} \mathrm{M}_{\odot}$.

Spectroscopy using HST and NOT indicate resonance absorption within the ejecta in the FUV with blue shifts of -1000 to $-3000 \mathrm{~km} \mathrm{~s}^{-1}$ (De Gennaro Aquino et al. 2014). After day $105 \mathrm{Fe}$ curtain turned transparent, but all absorption components persisted from day 105 to day 834 (see Figure 3 ). This is consistent with an outwardly moving ionization front following visual maximum after which the ejecta became optically thin and the ionization stages froze due to expansion. During nebular stages data is consistent with ballistically ejected shell with $\mathrm{n}_{e} \sim \mathrm{t}^{-3}$ (Shore et al. 2013, De Gennaro Aquino et al. 2014). In the nebular stage, ejecta can be modeled with axisymmetric conical bipolar geometry and a low inclination $\mathrm{i}=15 \pm 5$ degrees.

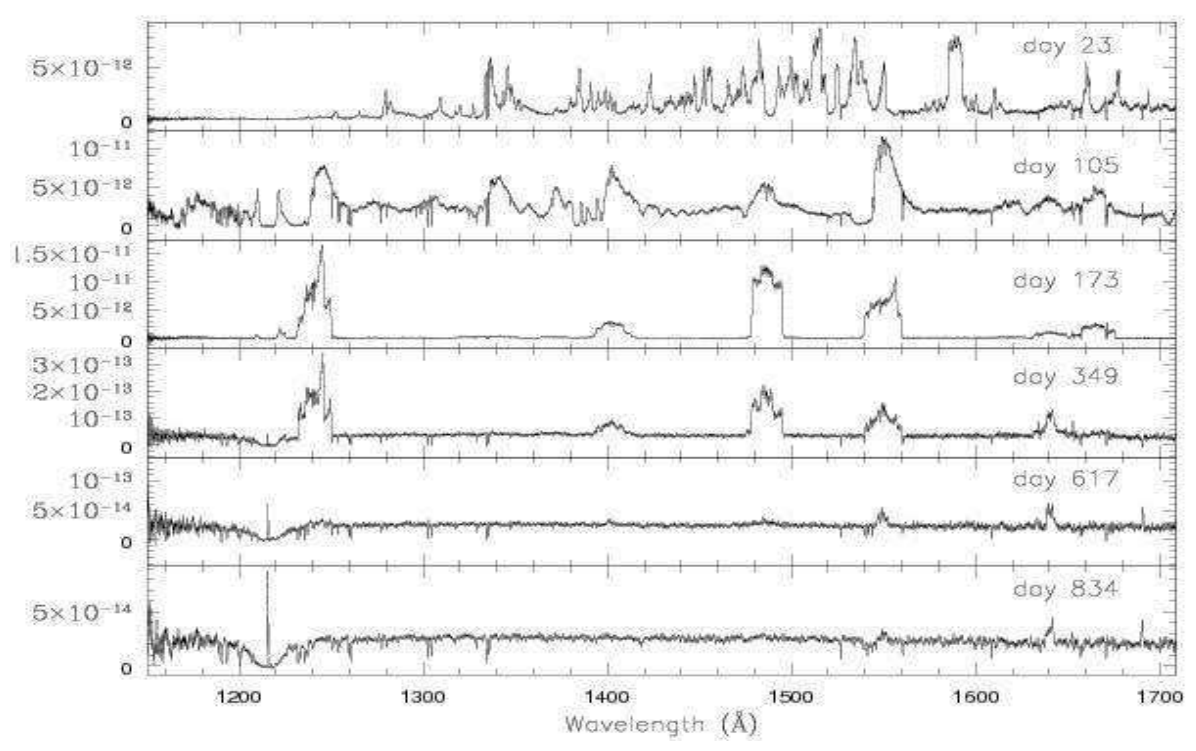

Figure 3: The UV spectral evolution of T Pyx in the 2011 outburst observed by NOT and HST (De Gennaro Aquino et al. 2014).

A face-on bipolar nebula is detected in NIR (Chesneau et al. 2011). Eruptions seems to have 
heated-up dust in the pre-existing nebulosity around T Pyx. The IR emission is consistent with interstellar dust swept up by T Pyx (rather than dust produced during eruptions) (Evans et al. 2012: SPITZER, Herschel).

\subsubsection{Radio and X-ray observations}

Radio observations (see Figure 4) have been conducted with JVLA (see Nelson et al. 2014 for details). The light curves and spectra show thermal emission (in the radio) from $\mathrm{T}$ Pyx during the entire outburst phase. In the first 50 days, there is cold and/or slowly expanding material with a radio spectral index $\alpha=1.8-1.4$. The optically thick thermal emission becomes partially optically thick by day 194 which changes to an optically thin shell emission with $\alpha=-0.23$ by day 300 . The radio results indicate an ejecta mass $\mathrm{M}_{e j}=(1-30) \times 10^{-5} \mathrm{M}_{\odot}$ for a range of filling factors and geometries. This suggests a sub-Chandrasekhar mass WD for T Pyx.

The Suzaku and Swift X-ray observations of T Pyx are summarized in Chomiuk et al. (2014)(see also Figure 4 for the Swift light curve). There is no detection of X-rays from T Pyx in the first 4 months till after 115 days after outburst with a late turn-on time. X-ray follow-up observations indicate a large ejecta mass of about $10^{-5} \mathrm{M}_{\odot}$. The soft X-ray component shows the highest temperature around $45 \mathrm{eV}$ revealing a $1 \mathrm{M}_{\odot} \mathrm{WD}$. The hard X-ray component has most temperatures around $1 \mathrm{keV}$. The X-ray light curve indicates a dual-phase ejection with large delay in between (2 months) consistent with radio observations. The high resolution Chandra and XMM-Newton data analysis (see Tofflemire et al. 2013) show a hard component which is found to be a plasma in collisional equilibrium with broadening in the lines of about 2000-3000 $\mathrm{km} \mathrm{s}^{-1}$. The soft component is detected by day 210 and 235 after outburst with a temperature of $420000 \mathrm{~K}(35 \mathrm{eV})$ partially obscured by the ejecta. Line ratios reveal electron densities of about $3 \times 10^{10} \mathrm{~cm}^{-3}$ with $\mathrm{T}_{e}>$ $2.5 \times 10^{6} \mathrm{~K}$. Figure 4 shows (on the right) the hard component (top) and soft component (bottom) of the X-ray spectrum of T Pyx obtained by XMM-Newton RGS and EPIC pn, respectively. Tofflemire et al. (2013), also, recovers the orbital period in the soft X-rays during the outburst phase, but the mean profile over the orbital phase is different than in quiescence (see Balman 2014). This may indicate existence of accretion and an accretion disk.

\subsection{Modeling of the old remnant and the Extended X-ray emission}

HST images of the ejecta surrounding the nova T Pyxidis resolve the emission into more than 2000 bright knots. Toraskar et al. (2013) simulate the dynamical evolution of the ejecta from T Pyxidis during its multiple eruptions over the last 150 years using the adaptive mesh refinement code Ramses. They demonstrate that the observed knots are the result of Richtmyer-Meshkov gas dynamical instabilities (Rayleigh-Taylor instabilities in an accelerated medium). These instabilities are caused by the overrunning of the ejecta from the classical nova of 1866 by fast-moving ejecta from the six subsequent recurrent nova outbursts. The model also predicts filamentary structure connecting the knots. The calculations indicate a possible X-ray luminosity of a few $\times 10^{29} \mathrm{erg} \mathrm{s}^{-1}$ from the older remnant around 5 arc sec radius.

A low $\mathrm{S} / \mathrm{N}(\sim 4-5 \sigma)$ extended excess emission was recovered in the XMM-Newton data of T Pyx (Balman 2010). In order to assess this better, a long observation of T Pyx was obtained in quiescence (in February 2011) with a much better spatial resolution capability using the Chandra ACIS-S detector (see Balman 2014 for observation details). The central source emission and its 

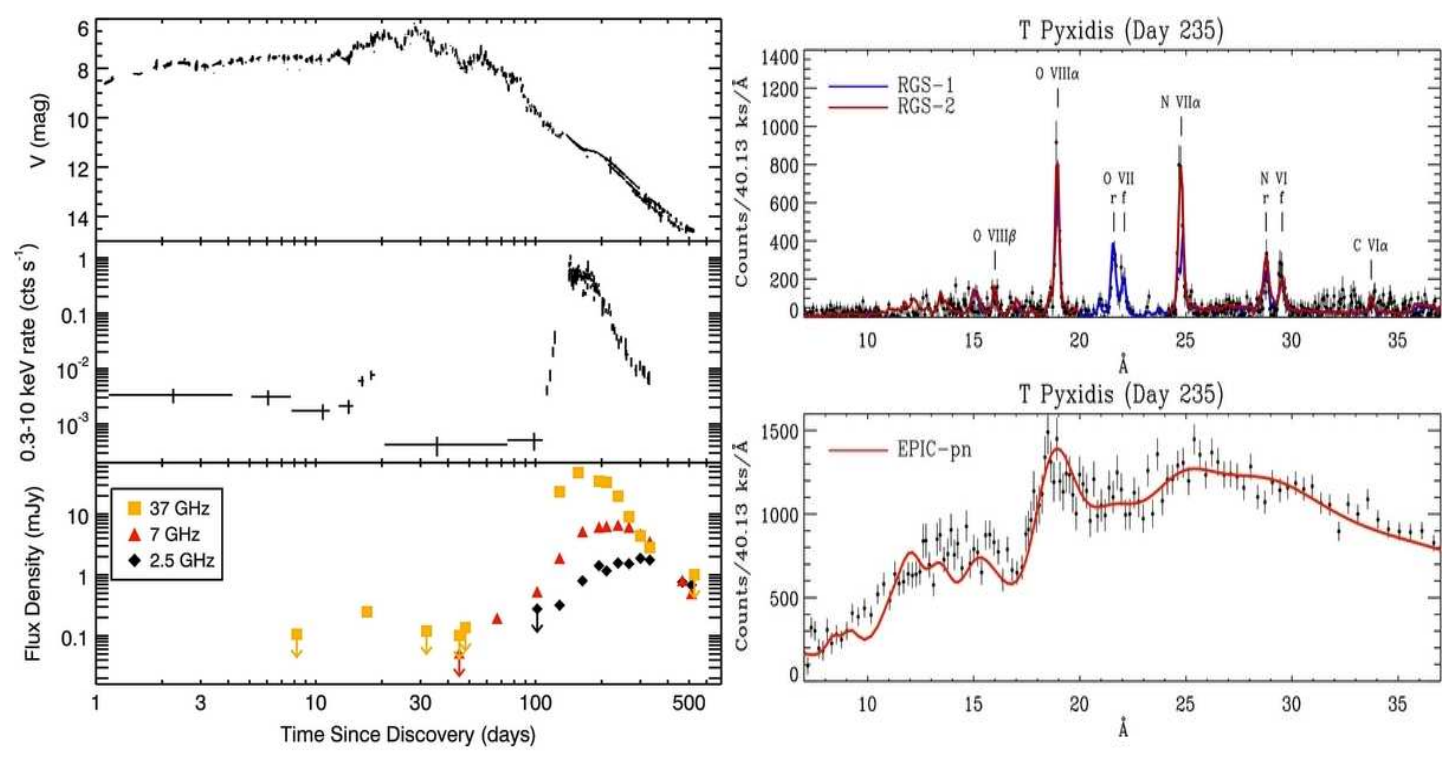

Figure 4: Left hand panel is the V-mag, AAVSO, X-ray, Swift, and radio, JVLA light curves (from top to bottom) of T Pyx obtained in the 2011 outburst (Chomiuk et al. 2014). Right panel displays the XMMNewton RGS (top) and EPIC pn (bottom) spectra (Tofflemire et al. 2013).

spectrum is deconvolved from any possible extended emission with a long and detailed highresolution imaging analysis (via smoothing and iterative PSF subtraction) at the subpixel level revealing an extended emission with $\mathrm{S} / \mathrm{N} \sim 6-10$. The derived shape looks like an elliptical nebula with a semi-major axis $\sim 1.0$ arc sec and a semi-minor axis $\sim 0.5$ arc sec, also indicating an elongation towards south (see Figure 5). The north-south inclination angle of the elliptical nebula is $i \leq 27^{\circ}$. The calculated approximate count rate of the extended emission is $0.0013-0.0025 \mathrm{c} \mathrm{s}^{-1}$. The nebular flux and luminosity are $(0.3-10.0) \times 10^{-14} \mathrm{erg} \mathrm{s}^{-1} \mathrm{~cm}^{-2}$ and $(0.6-30) \times 10^{31} \mathrm{erg} \mathrm{s}^{-1}$ (at $3.5 \mathrm{kpc}$ ) with considerable uncertainty; correct at the lower end of the ranges. The nebulosity seems consistent with an interaction of the outflow/ejecta from the 1966 outburst. The size of the torus-like structure $\sim 1^{\prime \prime}$ (a possible minimum due to projection effects) yields an expansion velocity of $V_{\exp } \geq 400 \mathrm{D}_{3.5 \mathrm{kpc}} \mathrm{km} \mathrm{s}^{-1}$.

Spectrum of the nebula is derived by a method involving creating energy-channel based images and performing PSF subtraction. The extended emission excess shows emission from plasma in ionization equilibrium or close to equilibrium with plausibly two temperatures around $0.6 \mathrm{keV}$ and $2.2 \mathrm{keV}$ with $\mathrm{N}_{H}=(0.2-0.9) \times 10^{22} \mathrm{~cm}^{2}$ (see Figure 5 right hand panel for an example). I note that the second harder X-ray temperature component has caveats from the spectral deconvolution process. Earlier work on modeling T Pyx shell and its interactions by Contini \& Prialnik (1997) yields that the circumstellar interaction of the T Pyx shells forms a forward shock that moves into the older ejecta and a reverse shock moves into the newer ejecta. The model predicts a faster, hotter, and denser reverse shock than the forward shock. Chandra results are in reasonable agreement with the predictions in this paper indicating a more recent interaction than assumed by Toraskar et al. (2013). The shocked mass in the X-ray nebula of T Pyx can be approximated as $\leq 1.8 \times 10^{-5} \mathrm{M}_{\odot}$ assuming a fully ionized gas, and $\mathrm{M}_{\text {neb }} \simeq \mathrm{n}_{\mathrm{e}} \mathrm{m}_{\mathrm{H}} \mathrm{V}_{\text {eff. }}$. Figure 6 shows a set of surface-brightness 
radial profiles indicating the existence of the extended emission. The profiles are obtained using a sector angle of 30 degrees centered on the north, south, east and west directions.
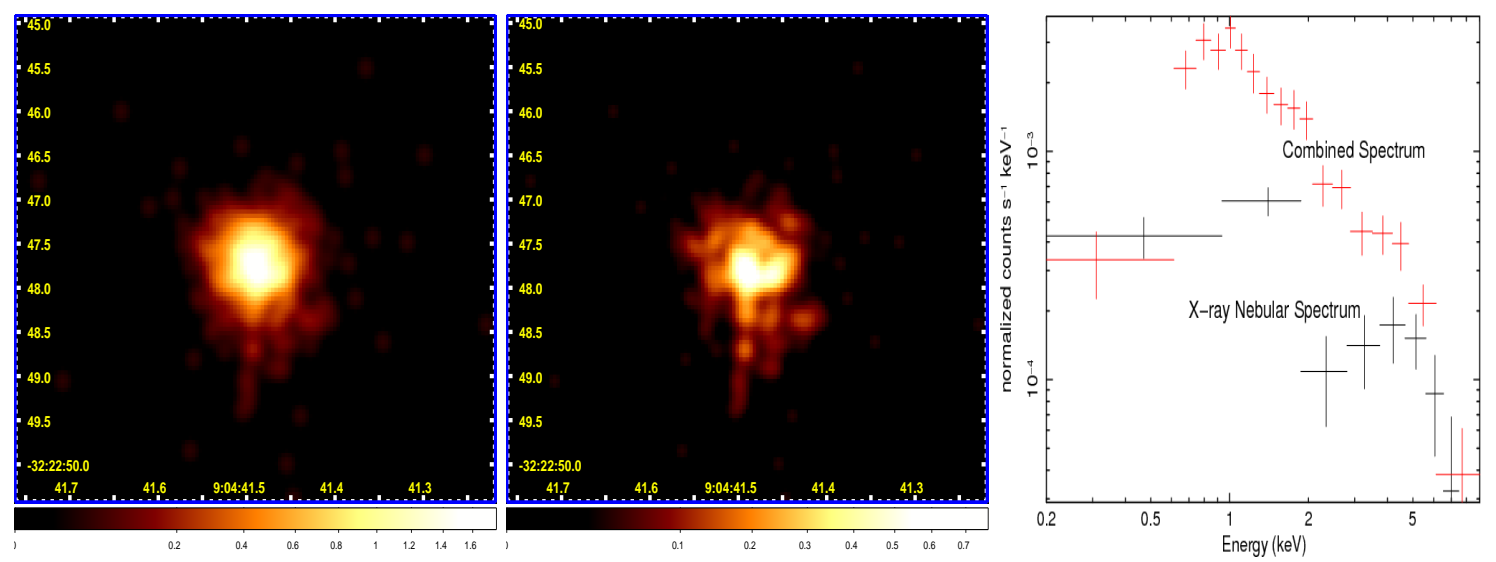

Figure 5: The high resolution image of T Pyx is on the left. North is up and west is to the right. The PSF subtracted image showing the extended nebulosity with semi-major axis about 1 arc sec is in the middle; the resolution is $0^{\prime \prime} .25$ per pixel (for details of the analysis see Balman 2014). The spectra on the right is of the point source (in red color) and the X-ray nebula (in black color). The data is obtained with Chandra during quiescence in 2011.
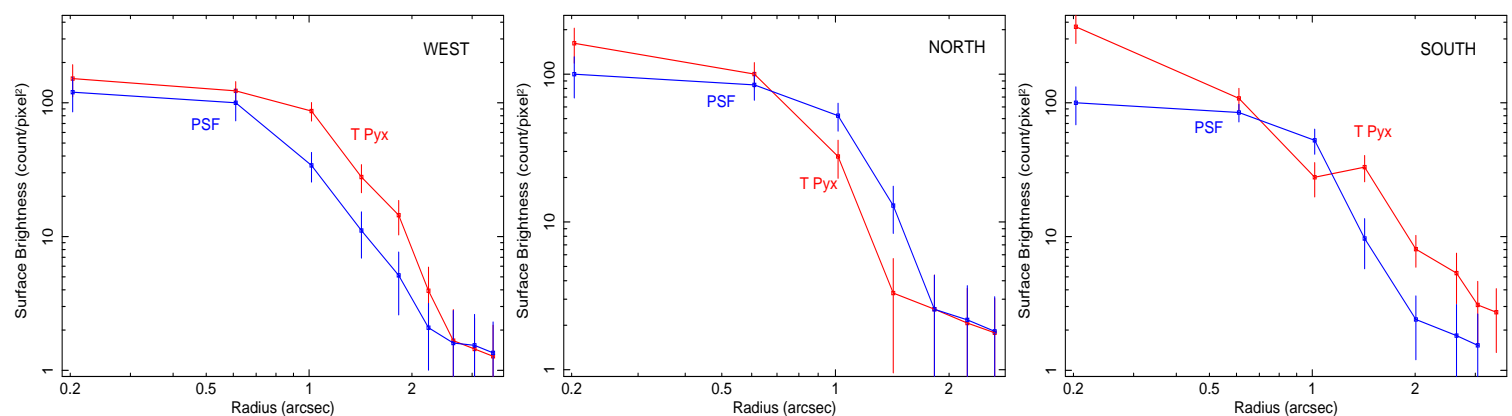

Figure 6: The radial profiles of surface brightness obtained towards different directions around T Pyx using a sector with 30 degrees opening angle (for details of analysis see Balman 2014). The Chandra data obtained during quiescence in 2011 is used.

\section{T Pyxidis - The binary system}

The spectroscopic period of T Pyx is Porb $=1.8295 \mathrm{~h}(\mathrm{f}=13.118368(11) \mathrm{Hz}$ ) with $\mathrm{K} 1=17.9(1.6)$ $\mathrm{km} \mathrm{s}^{-1} \mathrm{q}=0.2(3), \mathrm{M} 2=0.14(3) \mathrm{M}_{\odot}$, and $\mathrm{M} 1=0.7(2) \mathrm{M}_{\odot}$ (Uthas et al. 2010). The column density of hydrogen towards the source is consistent with $\mathrm{E}(\mathrm{B}-\mathrm{V})=0.25 \pm 0.02$ in quiescence (Schaefer et al. 2013) and it goes out to 0.5 in outburst (Shore et al. 2011). T Pyx is known for its very blue color in the optical band (see Figure 7 left hand side) which was attributed to possible H-burning in quiescence (Webbink et al. 1987, Patterson et al. 1998, Knigge et al. 2000).

The spectral energy distribution (SED) is dominated by an accretion disk in the UV+opt+IR ranges, with a power law distribution $F_{\lambda}=4.28 \times 10^{-6} \lambda^{-2.33} \mathrm{erg} \mathrm{s}^{-1} \mathrm{~cm}^{-2}$ per angstrom (after correction for reddening), while the continuum in the UV range can also be represented by a single 
blackbody of T $\sim 34,000 \mathrm{~K}$ with $\dot{M} \sim(1-4) \times 10^{-8} \mathrm{M}_{\odot} \mathrm{yr}^{-1}$ and $\mathrm{L}_{\text {disk }}$ about $3 \times 10^{35}$ erg s${ }^{-1}$ (for 0.7$1.4 \mathrm{M}_{\odot}$ WD) using 16 years of IUE data which remained constant in slope and intensity (Gilmozzi \& Selvelli 2007; Selvelli et al. 2008). Therefore, T Pyx is believed to be a nonmagnetic CV accreting at high rates as expected from RN precursors with a distance estimate of $3.50 \pm 0.35 \mathrm{kpc}$ (Selvelli et al. 2008). Recently, the ultraviolet-optical-infrared spectral energy distribution is found to be fitted by a power law $\left(f_{v} \propto v^{1}\right)$ which suggests that most of the T Pyx light in quiescence may not originate from a standard accretion disk, or any superposition of blackbodies, but rather comes from some nonthermal source (Schaefer et al. 2013). Furthermore, some studies in the optical and UV have yielded mass accretion rates of (1-10) $\times 10^{-7} \mathrm{M}_{\odot} \mathrm{yr}^{-1}$ calculated using optical B mags (Schaefer et al. 2013, Patterson et al. 2014) assuming the blue flux is proportional to accretion rate during quiescence and UV spectra (Godon et al. 2014) with the same assumption as above. Schaefer et al. (2013) clearly indicates flattening (in the UBVRIJHK SED; see Figure 7 right hand panel) from the main stream $f_{v} \propto v^{2}$ and $f_{v} \propto v^{0.3}$ dependencies which actually reveals divergence from standard disk accretion flows rather than existence of a nonthermal source which is not a disk as interpreted in this paper. Orbital period is found to be increasing $\mathrm{P} / \dot{P}=313000$ yr with (1.73.5) $\times 10^{-7} \mathrm{M}_{\odot} \mathrm{yr}^{-1}$ if the blue flux is proportional to accretion rate during quiescence. During outburst $\mathrm{P} / \dot{P}=300000 \mathrm{yr}$ and $\Delta$ Porb $=0.0054(6)$ leading to $\mathrm{M}_{e j}=3 \times 10^{-5} \mathrm{M}_{\odot}$ (Patterson et al. 2014).
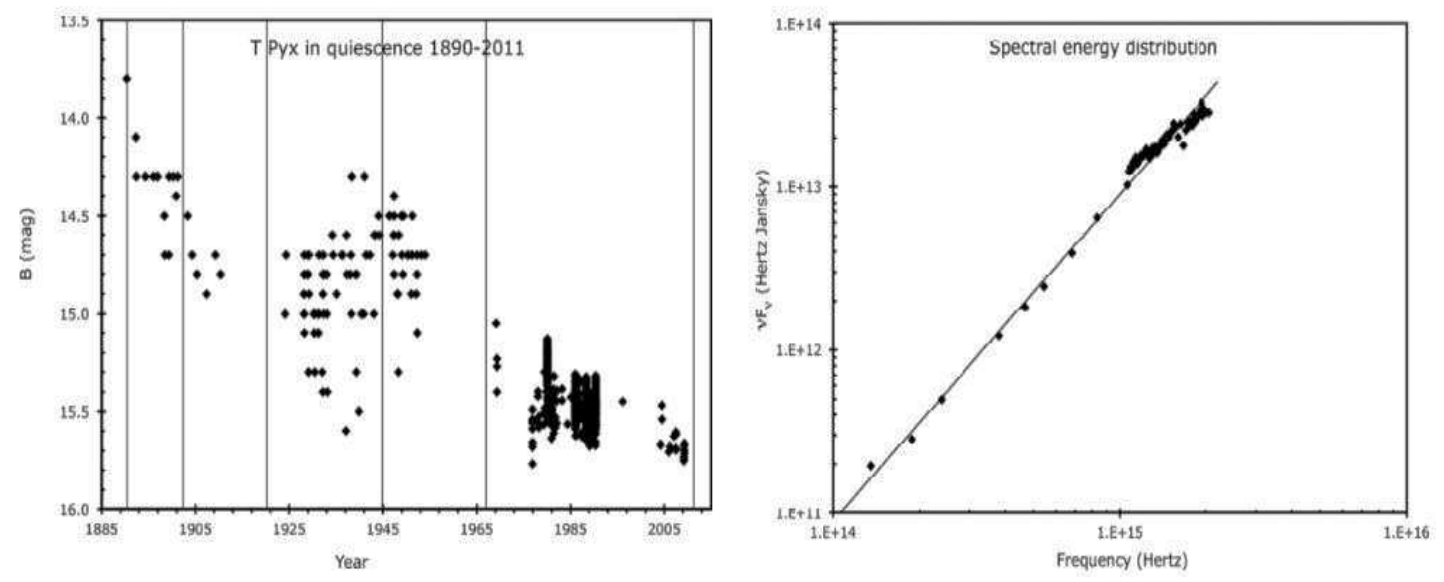

Figure 7: On the left, the B-band magnitude history of T Pyx is given from all existing data (see Schaefer et al. 2013). The solid vertical lines are times of recurrent nova outbursts. The right hand side shows the UBVRIJHK SED together with the UV GALEX data fitted with a power law of $f_{v} \propto v^{1}$ (Schaefer et al. 2013).

\subsection{The quiescent $\mathrm{X}$-ray emission}

The latest quiescent observations of T Pyx were obtained 2-3 months before its outburst in April 2011 (see Balman 2014 for a detailed review of the existing data) using the Chandra Observatory ACIS-S detector. The total source spectrum (see Figure 8) is consistent with a multitemperature (distribution) thermal plasma emission model (e.g., CEVMKL) (rather than a single temperature thermal plasma (e.g., MEKAL) or a single power-law model) with a maximum temperature $k T_{\max }>47.0 \mathrm{keV}(2 \sigma$ lower limit is $37 \mathrm{keV})$, and an unabsorbed X-ray flux of (0.9$1.5) \times 10^{-13} \mathrm{erg} \mathrm{s}^{-1} \mathrm{~cm}^{-2}$ with an X-ray luminosity $(1.3-2.2) \times 10^{32} \mathrm{erg} \mathrm{s}^{-1}$ in the $0.1-50.0 \mathrm{keV}$. I 
note that a more detailed modeling and comparisons with other models can be found in Balman (2014). The maximum plasma temperatures are virialized and thus, the plasma is likely not confined to the disk. The absorption towards the source is at the interstellar level consistent with the $\mathrm{E}(\mathrm{B}-\mathrm{V})$ values as determined from the X-ray fits. The binary period is also detected in the X-ray data (see Figure 8, right hand panel). The standard disk theory at steady state with constant $\dot{M}$ predicts, an optically thick boundary layer at the accretion rate of T Pyx $\left(\dot{\mathrm{M}} \geq \times 10^{-8} \mathrm{M}_{\odot} \mathrm{yr}^{-1}\right)$, with a blackbody emission in the soft X-ray/EUV regime ( Popham \& Narayan 1995; Godon et al. 1995). These models predict optically thick BLs with blackbody temperatures of 13-33 eV and $L_{\text {soft }} \geq 1 \times 10^{34}$ for $0.8-1.0 \mathrm{M}_{\odot} \mathrm{WD}$ (a rotation as high as $\Omega_{*}=0.5 \Omega_{K}\left(R_{*}\right)$ is already assumed in luminosity/temperature limits). I find no such emission with a $2 \sigma$ upper limit to the blackbody temperature as $k T_{\mathrm{BB}}<25 \mathrm{eV}$ and unabsorbed flux as $F_{\mathrm{BB}}<1.5 \times 10^{-12} \mathrm{erg} \mathrm{s}^{-1} \mathrm{~cm}^{-2}$ at $L_{\mathrm{BB}}<2.0 \times 10^{33}$ $\mathrm{erg} \mathrm{s}^{-1}(0.1-10.0 \mathrm{keV})$. This result is consistent with all such results attained from the X-ray wavelengths in time. I emphasize that these upper limits on temperature and flux is particularly not in accordance with the deep gravitational potential well of a near-Chandrasekhar WD for production of boundary layer emission from a rotating WD as high as $\Omega_{*}=0.5 \Omega_{K}\left(R_{*}\right)$ (see Popham \& Narayan 1995, Hertfelder et al. 2013). Moreover, these upper limits do not confirm any SSS emission from a suggested H-burning WD during quiescence and constrains the maximum accretion rate that can be attained $\left(\dot{\mathrm{M}}<1 \times 10^{-7} \mathrm{M}_{\odot} \mathrm{yr}^{-1}\right)$. In addition, I note that $\mathrm{T}$ Pyx does not have detected wind emission (see Uthas et al. 2010).
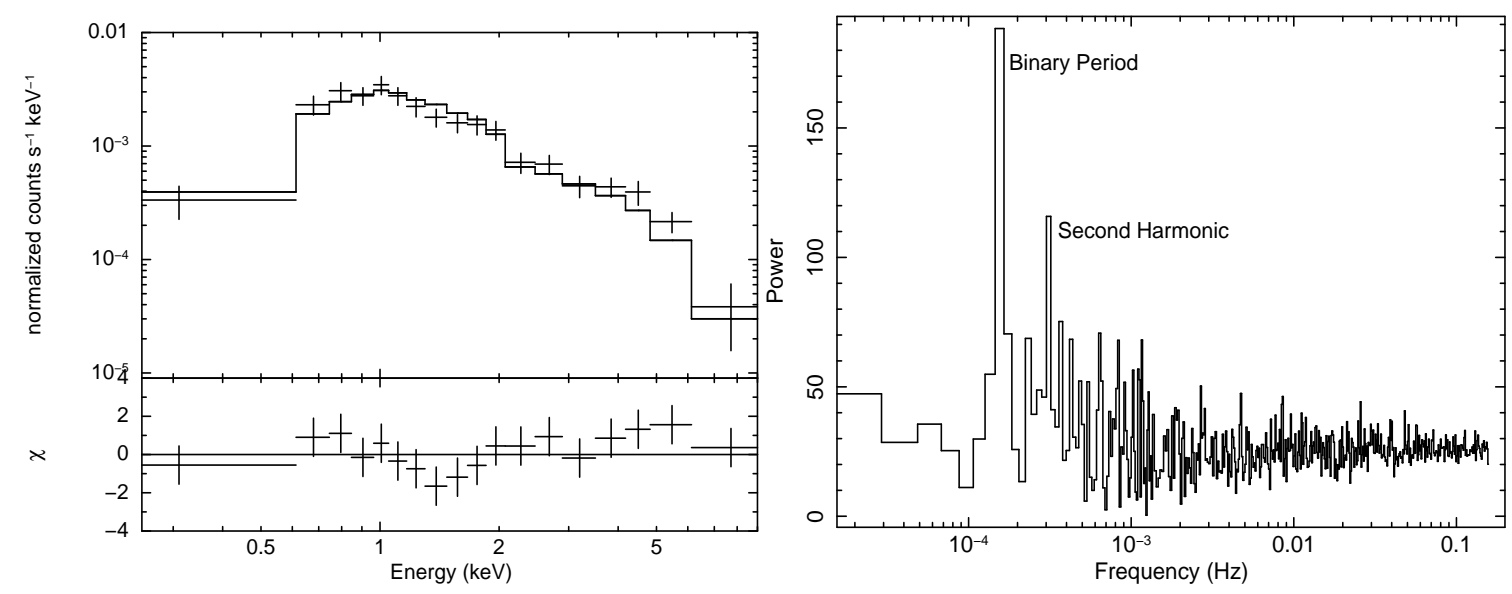

Figure 8: Left hand panel is the total X-ray spectrum of $\mathrm{T}$ Pyx in quiescence obtained with Chandra Observatory (Balman 2014). The right hand panel is the power spectrum of the light curve from the same data set showing the orbital period.

\subsection{Origin of the optically thin emission}

Detailed calculations by Narayan \& Popham (1993) show that the optically thin BLs of accreting WDs in CVs can be radially extended and that they advect part of the internally dissipated energy as a consequence of their inability to cool, therefore indicating that optically thin BLs act like advective hot accretion flows (i.e., ADAF-like: advection-dominated accretion flow). In addition, Popham \& Narayan (1995) illustrates that the BL can stay optically thin even at high accretion rates (as for T Pyx) for optical depth $\tau<1$ together with $\alpha \geq 0.1$ (disk viscosity $v=\alpha \mathrm{c}_{s} \mathrm{H}$ where 
$\mathrm{c}_{s}$ is the sound speed, $\mathrm{H}$ is the disk height and $\alpha$ is a free parameter in a range $0-1$ ). However, nature of such models have not been well investigated. An ADAF around a WD can be described by truncating the ADAF solutions at the WD surface as opposed to BHs and the accretion energy is advected onto the WD heating it up. Medvedev \& Menou (2002) and Menou (2000) include some preliminary work regarding ADAF-like flows and hot settling flows in CVs (dwarf novae) where Menou (2000) suggests that ADAF-like gas flows in the BLs of CVs can be one-temperature flows since Coulomb interactions are independent of advection quality of the accretion flow. Recently, Balman et al. (2014) have shown that some Nova-like CVs have BLs that can be characterized with ADAF-like flows merged with optically thin BLs in high state CVs. These objects have accretion rates similar to or somewhat less than $\mathrm{T}$ Pyx. The $\mathrm{X}$-rays have optically thin multiple-temperature cooling flow type emission spectra with temperatures $k T_{\max }$ in a range $21-50 \mathrm{keV}$ (see Figure 9, left hand panel). These BL regions are also found to be divergent from the isobaric cooling flow models and the temperatures are at/around virial values with similar characteristics to quiescent X-ray emission of T Pyx. The WD temperatures in these systems are around 45000-50000 K indicating advective heating is possible. Balman et al. (2014) estimates that the WD luminosity ratios using these temperatures compared with the low temperatures of the WDs in magnetic Polar systems (without disks) can consistently explain the inefficiency of the luminosity of the boundary layers in these nova-likes.

Given the disk luminosity mentioned previously for T Pyx and the X-ray luminosity in the $0.1-50 \mathrm{keV}$ range, their ratio is $\left(L_{\mathrm{X}} / L_{\mathrm{disk}}\right) \simeq(2-7) \times 10^{-4}$. The nature of the boundary layer in the central source of T Pyx is not consistent with the predictions from the calculations of the standard steady-state disk models $\left(L_{\mathrm{x}} \sim L_{\text {disk }}\right)$ (e.g., Narayan \& Popham 1993, Popham \& Narayan 1995). A $\left.L_{\mathrm{x}} / L_{\text {disk }}\right) \simeq 0.01-0.001$ was recovered for some nova-like systems in Balman et al. (2014). I note that discrepancy between $L_{\mathrm{x}}$ and $L_{\mathrm{disk}}$ is common for nonmagnetic CVs and this ratio has been found to be around 0.1 for SU UMa-type dwarf novae and about 0.01 for the U Gem sub-type together with the nova-likes at high states having a ratio of around 0.001 (see Kuulkers et al. 2006 for a review). In X-ray binaries, particularly black hole systems, an inner advection-dominated accretion flow exists that extends from the black hole horizon to a transition radius and above the disk there is a hot corona which is a continuation of the inner ADAF (Esin, McClintock \& Narayan 1997, Narayan $\&$ McClintock 2008). ADAFs are based on $\alpha$-viscosity prescription where substantial fraction of the viscously dissipated energy is stored in the gas and advected to the central object with the accretion flow rather than being radiated. This may explain the orders of magnitude difference in the X-ray luminosity in comparison with the accretion luminosity in the UV/optical bands for T Pyx. Such advective flows are referred as RIAF ADAFs (radiation inefficient accretion flows).

The flatter SED in the optical and UV for T Pyx is readily observed in Black Hole binaries and AGN disks as a direct indication of irradiation of the accretion disk with a nonstandard warped disk structure. For example, LMXB BH systems have optical+UV+IR SEDs with $f \propto v^{0.5-1.5}$ (Hynes 2005, Cadolle Bel et al. 2007, Zurita-Heras et al. 2011). QSO and AGN SEDs show similar behavior with even flatter indices (Francis et al. 1991). Optically thick disk truncation in dwarf novae is also an evidence for formation of hot flows in the inner parts of the CV disks. These are detected in the X-ray, some in the UV and optical power spectra of the light curves with the break frequencies in a range 1-6 $\mathrm{mHz}$ yielding truncation radii in a range $(3-10) \times 10^{9} \mathrm{~cm}$ (see Figure 9; Balman \& Revnivtsev 2012, Balman 2015 and references therein). 

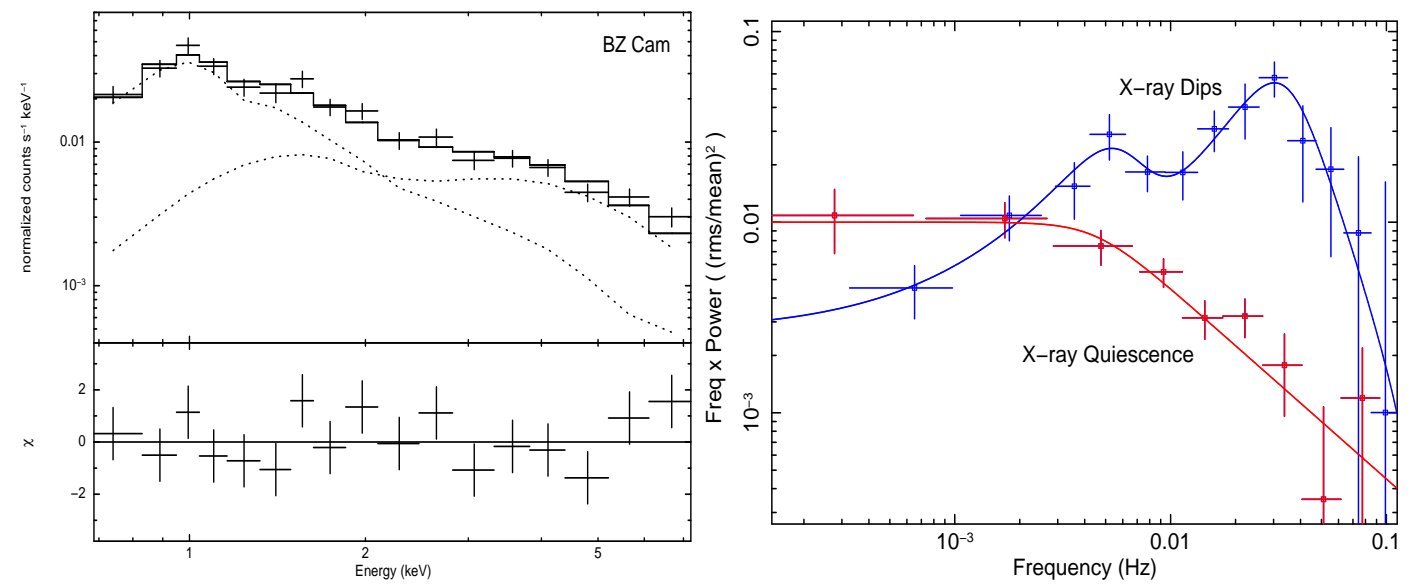

Figure 9: The Swift X-ray spectrum of a Nova-like system, BZ Cam (Balman et al. 2014) is on the left hand panel. On the right is the PDS of SS Cyg obtained using RXTE data in the X-ray wavelengths in quiescence (red color) and outburst (blue color) as indicated on the panel (Balman \& Revnivtsev 2012).

\section{Discussion and Conclusions}

Overall, T Pyx is a moderately fast nova that ejects about $3 \times 10^{-5} \mathrm{M}_{\odot}$ in very eruption with about 20 to $40 \mathrm{yr}$ recurrence time. The mass of the WD in the system is $0.7-1.0 \mathrm{M}_{\odot}$. It has some associated elliptical X-ray nebulosity with a semi-major axis about 1 arc sec.

The X-ray observations of T Pyx obtained in the quiescent phase, 2-3 months before its outburst in April 2011 indicate that the total source spectrum is consistent with a multi-temperature thermal plasma emission model with a maximum temperature $k T_{\max }>47.0 \mathrm{keV}$ ( $2 \sigma$ lower limit is $37 \mathrm{keV})$. It has an unabsorbed X-ray flux of (0.9-1.5) $\times 10^{-13} \mathrm{erg} \mathrm{s}^{-1} \mathrm{~cm}^{-2}$ with an X-ray luminosity $(1.3-2.2) \times 10^{32} \mathrm{erg} \mathrm{s}^{-1}$ in the $0.1-50.0 \mathrm{keV}$. The maximum plasma temperatures are virialized. The absorption towards the source determined from X-ray emission in quiescence is at the interstellar level consistent with the $\mathrm{E}(\mathrm{B}-\mathrm{V})$.

The standard disk theory at steady state with constant $\dot{M}$ predicts, an optically thick boundary layer at the accretion rate of T Pyx $\left(\dot{\mathrm{M}} \geq \times 10^{-8} \mathrm{M}_{\odot} \mathrm{yr}^{-1}\right)$, with a blackbody emission in the soft $\mathrm{X}$-ray/EUV regime. The binary has never been detected as a Soft X-ray emitter or a Super Soft X-ray Source since 1998 including the new observations and the upper limits on any blackbody emission are $\mathrm{F}_{B B}<1.5 \times 10^{-12} \mathrm{erg} \mathrm{s}^{-1} \mathrm{~cm}^{-2} \mathrm{~L}_{B B}<2 \times 10^{33} \mathrm{erg} \mathrm{s}^{-1}(0.1-10.0 \mathrm{keV}), \mathrm{kT}_{B B}<25$ $\mathrm{eV}$, with $\dot{M}<1 \times 10^{-7} \mathrm{M}_{\odot} \mathrm{s}^{-1}$. I emphasize that these upper limits on temperature and flux is particularly not in accordance with the deep gravitational potential well of a near-Chandrasekhar WD in the context of RN for production of boundary layer emission from a rotating WD as high as $\Omega_{*}=0.5 \Omega_{K}\left(R_{*}\right)$. Thus, X-ray observations support an optically thin BL region in T Pyx merged with ADAF-like hot flows and/or accretion disk corona which heats the WD as well (with advective accretion). The efficiency of emission in the boundary layer is $\mathrm{L}_{x} / \mathrm{L}_{\text {disk }} \simeq(2-7) 1 \times 10^{-4}$.

Standard nova theory requires for recurrent novae, high accretion rates and near Chandrasekharmass WDs. However, thermonuclear runaways and the recurrence of the TNRs are strong functions of the WD temperature and luminosity as much as the quiescent accretion rate, WD mass, accreted mass, and metal abundances. An advected luminosity equivalent of a disk luminosity of $3 \times 10^{35}$ $\mathrm{erg} \mathrm{s}^{-1}$ as calculated for T Pyx, will yield a WD effective temperature of $2 \times 10^{5} \mathrm{~K}$ for a $1 \mathrm{M}_{\odot}$ 
WD, consistent with effective WD temperatures during the onset of TNRs (see Starrfield et al. 2000, 2012). Thus, a scenario of advective hot flow accretion onto a WD consistently explains sub-Chandrasekhar mass WD in T Pyx and the large ejecta mass detected in all RN explosions of this source together with the evolution of the T Pyx light curves that are more typically found in slow (or slower) novae associated with lower mass WDs.

Therefore, T Pyx exposes an uncharted area in the standard nova theory and CV accretion and is a new theoretical challenge than an enigma of observations.

\section{Acknowledgements}

SB acknowledges support from TUBITAK, The Scientific and Technological Research Council of Turkey, through project 114F351.

\section{References}

[1] Ş. Balman, 2015, AcPPP, 2, 116.

[2] Ş. Balman, P. Godon, E. Sion, 2014, ApJ, 794, 84.

[3] Ş. Balman, 2014, A\&A, 572A, 114.

[4] Ş. Balman, M. Revnivtsev, 2012, A\&A, 546, 112.

[5] Ş. Balman, 2012, Mem. S.A.It., 83, 585.

[6] Ş. Balman, P. Godon, E. M. Sion, J.-U. Ness, E. Schlegel et al., 2011, ApJ, 741, 84.

[7] Ş. Balman, 2010, MNRAS, 404, L26.

[8] M. F. Bode, and A. Evans, 2008, Classical Novae (Classical Novae, 2nd Edition. Edited by M.F. Bode and A. Evans. Cambridge Astrophysics Series, No. 43, (Cambridge: Cambridge University Press)

[9] M. F. Bode et al. 2006, ApJ, 652, 629.

[10] M. Cadolle Bel et al., 2007, A\&A, 659, 549.

[11] R. M. Catchpole, 1969, MNRAS, 142, 119.

[12] O. Chesneau et al., 2011, A\&A, 534, L11.

[13] L. Chomiuk et al., 2014, ApJ, 788, 130.

[14] M. Contini, D. Prialnik, 1997, ApJ, 475, 803.

[15] C. Done, M. Gierliński, A. Kubota, 2007, A\&ARv, 15, 1.

[16] A. Ederoclite, 2014, in Stella Novae: Past and Future Decades. ASP Conf. Ser., Vol. 490, P. A. Woudt, V. A. R. M. Ribeiro (eds.), p. 163.

[17] A. Esin, J. E. McClintock, R. Narayan, 1997, ApJ, 489, 805. 
[18] A. Evans, R. D. Gehrz, L. A. Helton, S. Starrfield, M. F. Bode et al., 2012, MNRAS, 424, L69.

[19] P. J. Francis, P. C. Hewett, C. B. Foltz, F. H. Chaffee, R. J. Weymann et al., 1991, ApJ, 373, 465.

[20] I. De Gennaro Aquino, S. N. Shore, G. J. Schwarz, E. Mason, S. Starrfield, E. M. Sion, 2014, $A \& A, \mathbf{5 6 2 A}, 28$.

[21] R. Gilmozzi, P. Selvelli, 2007, A\&A, 461, 593.

[22] P. Godon, O. Regev, G. Shaviv, 1995, MNRAS, 275, 1093.

[23] P. Godon, E. M. Sion, S. Starrfield, M. Livio, R. E. Williams, R.E. et al., 2014, ApJ, 784, L33.

[24] M. Hernanz, G. Sala, 2002, Science, 298, 393.

[25] M. Hertfelder, W. Kley, V. Suleimanov, K. Werner, 2013, A\&A, 560A, 56.

[26] R. I. Hynes, 2005, ApJ, 623, 1026.

[27] L. Izzo et al., 2012, Mem. S.A.It., 83, 830.

[28] K. Imamura, K. Tanabe, 2012, PASJ, 64, L9.

[29] C. Knigge, A. R. King, J. Patterson, 2000, A\&A, 364, L75.

[30] J. Krautter, 2008, in Classical Novae, (eds.) M. F. Bode \& A. Evans, Wiley, Cambridge University Press, second edition, Chapt. 10

[31] E. Kuulkers, A. Norton, A. Schwope, B. Warner, 2006, in Compact stellar X-ray sources, W. Lewin, M. van der Klis (eds.), Cambridge Astrophysics Series No. 39, (Cambridge, UK: Cambridge University Press), p. 421.

[32] D. Lynden-Bell, J. E. Pringle, 1974, MNRAS, 168, 603.

[33] M. Medvedev, K. Menou, 2002, ApJ, 565, 39.

[34] K. Menou, 2000, pre-print, (astro-ph/000785)

[35] R. Narayan, R. Popham, 1993, Nature, 362, 820.

[36] R. Narayan, J. E. McClintock, 2008, NewAR, 51, 733.

[37] T. Nelson, D. Donato, K. Mukai, J. Sokoloski, L. Chomiuk, 2012, ApJ, 748, 43.

[38] T. Nelson et al., 2014, ApJ, 785, 78.

[39] J.-U. Ness et al., 2009, AJ, 137, 3414.

[40] T. J. O'Brien, J. G. Cohen, 1998, ApJ, 498, L59.

[41] M. Orio et al., 2001, MNRAS, 326, L13. 
[42] K. L. Page et al., 2010, MNRAS, 401, 121.

[43] K. L. Page et al., 2015, MNRAS, 454, 3108.

[44] J. Patterson, J. Kemp, A. Shambrook et al., 1998, PASP, 110, 380.

[45] J. Patterson, A. Oksanen, B. Monard, R. Rea, F. Hambsch et al., 2014, in Stella Novae: Past and Future Decades. ASP Conf. Ser., Vol. 490, P. A. Woudt, V. A. R. M. Ribeiro (eds.), p.35.

[46] R. Popham, R. Narayan, 1995, ApJ, 442, 337.

[47] B. E. Schaefer, A. Pagnotta, M. M. Shara, 2010, ApJ, 708, 381.

[48] B. E. Schaefer et al., 2013, ApJ, 773, 55.

[49] B. E. Schaefer, 2010, ApJ, 187, 275.

[50] P. Selvelli, A. Cassatella, R. Gilmozzi, R. González-Riestra, 2008, A\&A, 492, 787.

[51] M M. Shara, 1989, PASP, 101, 5.

[52] M. M. Shara, A. Moffat, R. E. Williams, J. Cohen, 1989, ApJ, 337, 720.

[53] M. M. Shara, D. Zurek, R. E. Williams, D. Prialnik, R. Gilmozzi, A.F.J. Moffat, 1997, AJ, 114, 258.

[54] M. M. Shara et al., 2015, ApJ, 805, 148.

[55] S. N. Shore, T. Augusteijn, A. Ederoclite, H. Uthas, 2011, A\&A, 533, L8.

[56] S. N. Shore, G. J. Schwarz, I. De Gennaro Aquino, T. Augusteijn, F. M. Walter et al., 2013, $A \& A, 549 \mathrm{~A}, 140$.

[57] J. L. Sokoloski, A. P. Crotts, S. Lawrence, H. Uthas, 2013, ApJ, 770, L33.

[58] S. Starrfield, W. Sparks, J. W. Truran, M. C. Wiescher, 2000, ApJS, 127, 205.

[59] S. Starrfield, C. Iliadis, F. X. Timmes, W. R. Hix, W. D. Arnett, C. Meakin, W. M. Sparks, 2012, BASI, 40, 419.

[60] F. Surina, R. A. Hounsell, M. F. Bode, M. J. Darnley, D. J. Harman, F. M. Walter, 2014, AJ, 147, 107.

[61] B. M. Tofflemire, M. Orio, K. L. Page, J. P. Osborne, S. Ciroi, V. Cracco et al., 2013, ApJ, 779, 22.

[62] J. Toraskar, M.-M. Mac Low, M. M. Shara, D. R. Zurek, 2013, ApJ, 768, 48.

[63] H. Uthas, C. Knigge, D. Steeghs, 2010, MNRAS, 409, 237.

[64] E. Waagan et al., 2011, CBET, 2700, 1. 
[65] B. Warner, 1995, Cataclysmic Variable Stars, Cambridge Univ. Press, Cambridge

[66] R. F. Webbink, M. Livio, J. W. Truran, M. Orio, 1987, ApSS, 131, 493.

[67] J. A. Zurita Heras, S. Chaty, M. Cadolle Bel, L. Prat, 2011, MNRAS, 413, 235. 